\title{
Transfer of Cornus florida and C. kousa Simple Sequence Repeats to Selected Cornus (Cornaceae) Species
}

\author{
Phillip A. Wadl ${ }^{1}$ \\ Entomology and Plant Pathology Department, University of Tennessee, 2431 Joe Johnson Drive, \\ 205 Ellington Plant Science Building, Knoxville, TN 37996-4560
}

Xinwang Wang

Texas AgriLife Research and Extension Center, Texas A\&M University System, 17360 Coit Road, Dallas, $T X 75252$

John K. Moulton

Entomology and Plant Pathology Department, University of Tennessee, 2431 Joe Johnson Drive, 205 Ellington Plant Science Building, Knoxville, TN 37996-4560

\author{
Stan C. Hokanson \\ Department of Horticultural Science, University of Minnesota, 258 Alderman Hall, 1970 Folwell \\ Avenue, St. Paul, MN 55108 \\ John A. Skinner \\ Entomology and Plant Pathology Department, University of Tennessee, 2431 Joe Johnson Drive, \\ 205 Ellington Plant Science Building, Knoxville, TN 37996-4560
}

Timothy A. Rinehart

USDA-ARS Thad Cochran Southern Horticultural Laboratory, 810 Highway 26 West, Poplarville, MS 39470

Sandra M. Reed

USDA-ARS Floral and Nursery Plants Research Unit, US National Arboretum, Tennessee State

University Nursery Research Center, 472 Cadillac Lane, McMinnville, TN 37110

Vincent R. Pantalone

Department of Plant Sciences, University of Tennessee, 2431 Joe Johnson Drive, Knoxville, TN 37996-4561

\author{
Robert N. Trigiano \\ Entomology and Plant Pathology Department, University of Tennessee, 2431 Joe Johnson Drive, 205 \\ Ellington Plant Science Building, Knoxville, TN 37996-4560
}

\begin{abstract}
AdDiTIONAL INDEX wORDS. cross-species transferability, dogwood, microsatellites, polymorphic information content, SSR
AbSTRACt. Cross-species transferability of simple sequence repeats (SSRs) is common and allows SSRs isolated from one species to be applied to closely related species, increasing the use of previously isolated SSRs. The genus Cornus consists of 58 species that are ecologically and economically important. SSRs have previously been isolated from $C$. florida and $C$. kousa. In this study, 36 SSRs were tested on taxa from 18 Cornus species and hybrids for cross-species transferability and genetic diversity was calculated for each locus using polymorphism information content (PIC). Cross-species transferability of SSR loci was higher in more closely related species and PIC values were high. Evidence was found for conserved primer sites as determined by the amplification of SSR loci in the taxa examined. Polymerase chain reaction products were cloned and sequenced for three SSR loci (CF48, CF59, and CF124) and all individuals sequenced contained the appropriate repeat. Phylogenetic relationships of 14 Cornus species were inferred using nucleotide sequences of SSR locus CF48. The most parsimonious tree resulting from this analysis was in concordance with phylogenies based on $m a t K$ and internal transcribed spacer sequences. The SSR loci tested in this study will be useful in future breeding, population, and genetic studies within Cornus.
\end{abstract}

Received for publication 16 Mar. 2010. Accepted for publication 13 Apr. 2010. This work was supported by USDA Grant no. 58-6404-7-213 and the J. Frank Schmidt Family Charitable Foundation.

Mention of trade names or commercial products in this article is solely for the purpose of providing specific information and does not imply recommendation or endorsement by Texas A\&M University, University of Minnesota, University of Tennessee, or the U.S. Department of Agriculture.

${ }^{1}$ Corresponding author. E-mail: pwadl@utk.edu.
Simple sequence repeat (SSR), also called microsatellites, are sections of DNA consisting of tandemly repeated mono-, di-, tri, tetra-, or pentanucleotide units that occur in abundance within the genomes of most eukaryotes (Powell et al., 1996). They were first documented in humans (Hamada et al., 1982) and later isolated and characterized in five tropical tree species (Conduit and Hubbell, 1991). SSRs are polymerase chain 
reaction (PCR)based molecular markers valued for their abundant and uniform genome coverage, high levels of polymorphism, codominance, reproducibility, and ease of genotyping. However, unlike other molecular markers, isolation of SSRs involves methods that are expensive and laborious such as creation of a small insert genomic library, library screening, DNA sequencing of positive clones, primer design, and PCR optimization. Despite the development of highly efficient enrichment techniques for the isolation of SSRs (Wang et al., 2007), many laboratories do not have the resources or expertise needed for the isolation of new SSR loci. However, these same laboratories do have the expertise and resources to analyze previously developed SSRs. One way to overcome the isolation obstacle is to test PCR amplification of SSRs from source species in related species and genera. The ability to transfer SSRs from one species to another depends on the primer sites flanking SSR motifs being conserved between the taxa. Shortly after the discovery of SSRs in plants, conservation of primer sites was demonstrated in rice [Oryza sativa (Wu and Tanksley, 1993)], grape [Vitis vinifera (Thomas and Scott, 1993)], and Citrus species (Kijas et al., 1995) and the cross-species transfer of SSRs has since been demonstrated with many other genera.

The genus Cornus (Cornaceae) consists of 58 species of mostly shrubs and small trees that are widely distributed in the temperate and subtropical regions of North America (Xiang et al., 2006). Phylogenetic relationships, based on morphological and molecular data, within the genus have been controversial for almost a century (Xiang et al., 2006). Xiang et al. (2006) conducted an extensive study to resolve phylogenetic relationships using internal transcribed spacer (ITS) and matK sequencing analysis and an expanded morphological data matrix. Sampling consisted of taxa from Cornus of nearly all widely accepted extant species and multiple populations of widely distributed species. The phylogenetic analyses identified the following four major clades: big-bracted group; dwarf dogwoods; cornelian cherries; and the blue- or white-fruited group. These clades were congruent with inflorescence and fruit morphology.

The only major clade that does not have species native to North America is the cornelian cherries. Cornus species from the big-bracted group are important ornamental crops in the United States, whereas species from all four of the major clades provide food and shelter for wildlife (Jones, 2005; Mitchell et al., 1988). In addition, C. amomum (silky dogwood), $C$. florida (flowering dogwood), and C. racemosa (gray dogwood) are important in the stabilization and conservation of natural habitats in the eastern United States. Recently, C. mas (cornelian cherry) fruits were documented to contain high levels of antioxidants and antioxidant activity (Tural and Koca, 2008), which varied by genotype (Yilmaz et al., 2008).

The identification of four major clades in Cornus (Xiang et al., 2006) led us to believe that SSR loci isolated from two species within the big-bracted group might transfer to other Cornus species in all four of the major clades. The ability to transfer SSRs from C. florida and C. kousa to other Cornus species will play an important role in plant breeding, conservation studies, and investigating gene flow within the genus.

In this study, we used primers developed from $C$. florida (Wang et al., 2008) and C. kousa (Wadl et al., 2008) to detect SSR loci in selected species within Cornus. Specifically, we address the following questions: 1) Can the SSR primers developed for one species be used to amplify homologous loci of related species? 2) Does the cross-species transferability of SSR loci result from conservation of primer sites? 3) At what taxonomic level are these SSR primers potentially useful? We also present a phylogenetic tree of selected Cornus species based on SSR sequence data.

\section{Materials and Methods}

Plant material and DNA extraction. Forty-four Cornus genotypes representing 18 species and hybrids were selected for analysis (Table 1). Unopened flower buds or emerging leaves, which were not fully expanded, were collected and stored at $-80^{\circ} \mathrm{C}$ until genomic DNA was extracted from each of the taxa. Samples were ground in liquid nitrogen and DNA extracted using the DNeasy Plant DNA isolation kit (Qiagen, Valencia, CA). DNA was quantified with a ultraviolet-Vis spectrophotometer (ND-1000; NanoDrop Technologies, Wilmington, DE); DNA quality was determined using 2\% agarose gels stained with ethidium bromide and visualized in the 2000 Gel Documentation System (Bio-Rad, Hercules, CA).

AMplification of SIMPle SEQUence Repeat LOCI AND GENETIC DIVERSITY ANALYSES. Previously shown polymorphic primer pairs from 17 C. florida (Wang et al., 2008) and $19 C$. kousa SSR loci (Wadl et al., 2008) were selected and used to test the cross-species amplification on selected Cornus taxa. PCR amplification of the C. florida SSRs was completed using the following conditions: $10 \mu \mathrm{L}$ PCR reactions contained $2 \mathrm{ng}$

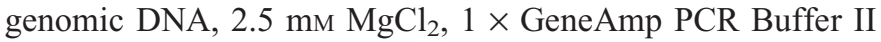
(Applied Biosystems, Foster City, CA), 0.2 mм dNTPs, 0.25 $\mu \mathrm{M}$ primer, 0.6 U AmpliTaq Gold ${ }^{\circledR}$ DNA polymerase (Applied Biosystems), and sterile, nanopure water. Cycling conditions were as follows: one cycle of $94^{\circ} \mathrm{C}$ for $5 \mathrm{~min}, 35$ cycles of $94{ }^{\circ} \mathrm{C}$ for $40 \mathrm{~s}, 55^{\circ} \mathrm{C}$ for $40 \mathrm{~s}, 72^{\circ} \mathrm{C}$ for $30 \mathrm{~s}$, and one cycle of $72{ }^{\circ} \mathrm{C}$ for 4 min. PCR amplification of the $C$. kousa SSRs was completed using the same components and cycling conditions listed for C. florida, except the annealing temperature was $58^{\circ} \mathrm{C}$. PCR products were sized on the QIAxcel Capillary Electrophoresis System (Qiagen) using an internal 25-bp DNA stepladder. To determine if the PCR products obtained from any PCR amplifications that produced faint or stutter bands were specific or nonspecific bands, the sample was reamplified by adding 0.5 $\mu \mathrm{L}$ of dimethyl sulfoxide [DMSO (Varadaraj and Skinner, 1994; Winship, 1989)] and using the same PCR components and conditions as previously mentioned.

Genetic diversity was determined for 17 C. florida SSR loci and 19 C. kousa SSR loci by calculating the polymorphism information content (PIC) (Anderson et al., 1992). This calculation was made using all individuals tested at each locus. When there was no amplification in a sample, with further confirmation, we assumed the presence of a null allele(s) and considered all null alleles to belong to a single allelic category.

Cloning AND SEQUeNCING OF SIMPLE SEQUENCE REPEAT LOCI. To confirm the conservation of primer sites and presence of SSR motifs, the PCR products amplified from selected Cornus species (Table 1) for three loci (CF48, CF59, and CF124) were cloned and sequenced. The PCR products were separated on $10 \%$ polyacrylamide gels, stained with silver (Bassam et al., 1991), and then excised from the gel and reamplified in $30-\mu \mathrm{L}$ PCR reactions containing $2.5 \mathrm{mM} \mathrm{MgCl}_{2}, 1 \times$ GeneAmp PCR Buffer II (Applied Biosystems), $0.2 \mathrm{~mm}$ dNTPs, $0.25 \mu \mathrm{M}$ primer, $1.8 \mathrm{U}$ AmpliTaq Gold ${ }^{\circledR}$ DNA polymerase (Applied Biosystems), and sterile, nanopure water. Cycling conditions 


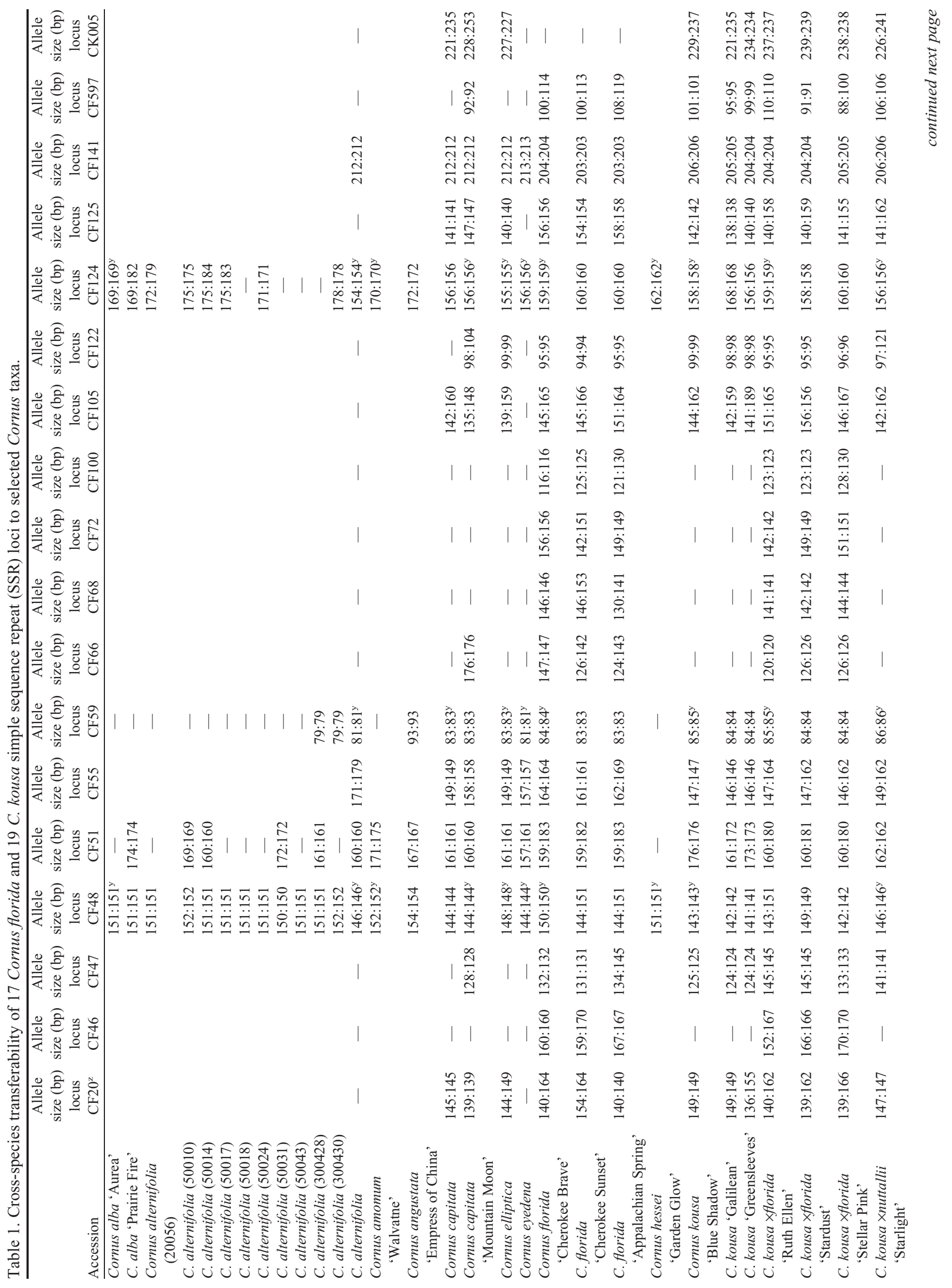




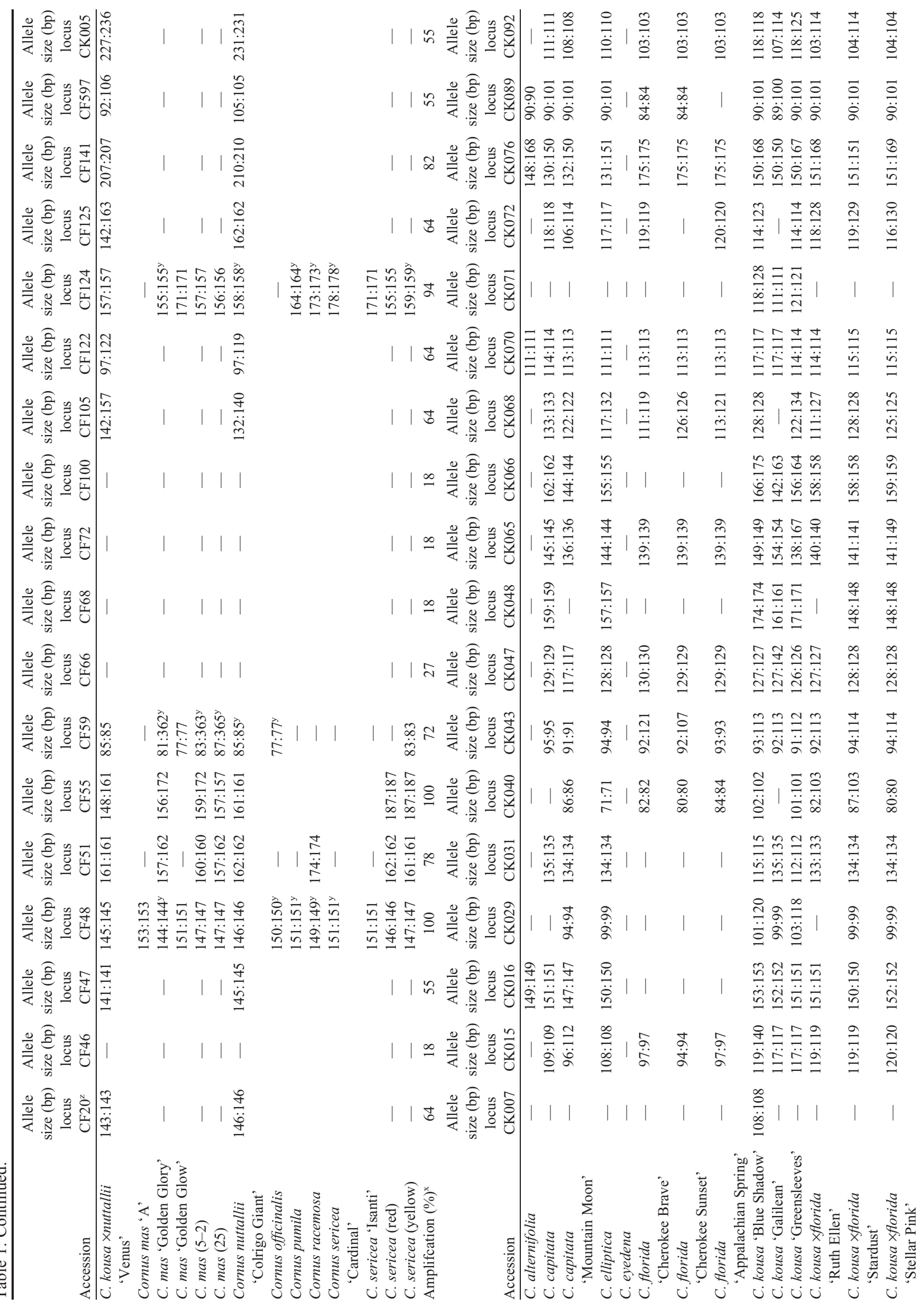




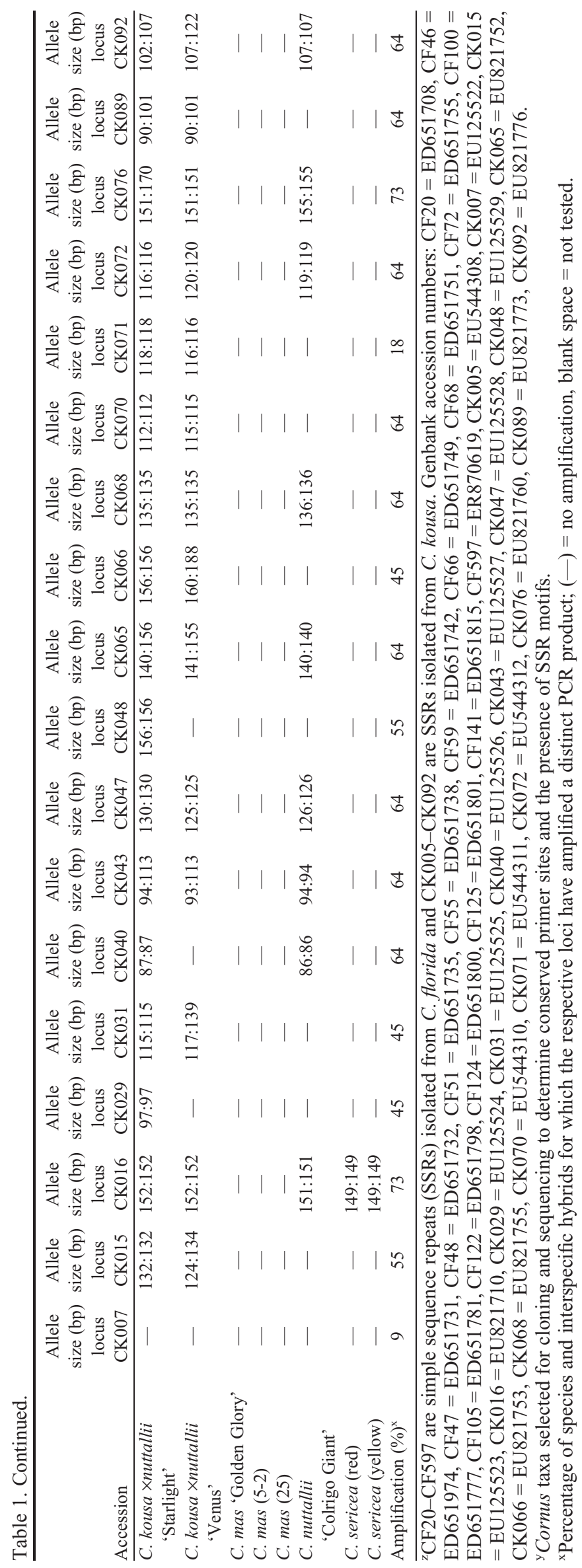

were as follows: one cycle of $94^{\circ} \mathrm{C}$ for $5 \mathrm{~min}, 35$ cycles of $94{ }^{\circ} \mathrm{C}$ for $40 \mathrm{~s}, 55^{\circ} \mathrm{C}$ for $40 \mathrm{~s}, 72^{\circ} \mathrm{C}$ for $30 \mathrm{~s}$, and one cycle of $72^{\circ} \mathrm{C}$ for 4 min. A QIAquick PCR Purification Kit (Qiagen) was used to eliminate primers, dNTPs, polymerases, and salts before cloning PCR products. The pGEM-T Easy Vector Systems (Promega, Madison, WI) was used for cloning. We followed the manufacturer's instructions except for the following modifications: our $10 \mu \mathrm{L}$ ligation reaction consisted of $2.5 \mu \mathrm{L}$ of $2 \times$ Rapid Ligation Buffer, $0.5 \mu \mathrm{L}$ pGEM-T Easy Vector, $3 \mu \mathrm{L}$ PCR product, $0.5 \mu \mathrm{L}$ T4 DNA ligase, and incubation overnight at $4{ }^{\circ} \mathrm{C}$. Our transformation reaction consisted of $4 \mu \mathrm{L}$ of ligation mixture, $15 \mu \mathrm{L}$ of JM109 High Efficiency Competent Cells (Promega), and $200 \mu \mathrm{L}$ instead of $950 \mu \mathrm{L}$ of SOC medium was used during the 1.5 -h incubation at $37^{\circ} \mathrm{C}$. To increase the number of colonies, the cells were allowed to settle in $1.5-\mathrm{mL}$ microcentrifuge tubes and $150 \mu \mathrm{L}$ of the SOC medium was removed from the tube and the remaining volume $(\approx 50 \mu \mathrm{L})$ was mixed gently, plated onto LB/ampicillin/IPTG/X-gal plates, and incubated at $37{ }^{\circ} \mathrm{C}$ overnight. Plasmid DNA was isolated using the QIAprep Miniprep Kit (Qiagen) and sequenced using ABI Big-Dye Version 3.1 terminators (Applied Biosystems). Sequencing reactions were cleaned using Centri-sep columns (Princeton Separations, Adelphia, NJ), electrophoresed through a 6\% polyacrylamide gel using an MJ Research BaseStation100 automated DNA sequencer (Bio-Rad), and analyzed using Cartographer 1.2.7 software (Bio-Rad). Sequences from opposing strands were reconciled and verified for accuracy using Sequencher 4.2.2 (Gene Codes, Ann Arbor, MI) and are archived in GenBank.

Phylogenetic analyses. Final sequences were aligned using Clustal X (Larkin et al., 2007) and analyzed phylogenetically. Nucleotides were subjected to maximum parsimony and Bayesian analysis. Parsimony searches used PAUP*'s heuristic search option with TBR branch rearrangement (Swofford, 2002). Characters were treated as unordered and maxtrees was set to increase incrementally. Most-parsimonious trees were found by conducting 1000 searches with each search begun from trees acquired by stepwise addition with a random addition sequence order. Bayesian analysis was conducted using MrBayes 3.1 (Ronquist and Huelsenbeck, 2003) using the best-fit model $(\mathrm{GTR}+\mathrm{I}+\mathrm{G})$ based on the AIC implemented in Modeltest (Posada and Crandall, 1998). Each Markov chain in the Bayesian search was started from a random tree and run for 2 million generations, sampling every 1000th cycle. To ensure that the inference was based on samples from the target distribution, the initial $25 \%$ of resulting trees was discarded as burn-in. Convergence was assessed by visual inspection of state likelihoods and average deviation of clade splits between replicate runs. Node support was evaluated by nonparametric bootstrap resampling (Felsenstein, 1985) and Bayesian posterior probabilities. Bootstrap scores for parsimony analyses were calculated from 1000 replicates with each replicate consisting of single searches starting with a tree built by stepwise addition using the simple addition sequence.

\section{Results}

CROSS-SPECIES AMPLIFICATION OF SIMPLE SEQUENCE REPEAT LOCI. A total of 36 loci were tested across 22 genotypes and four of the 36 loci (CF48, CF51, CF59, and CF124) were tested across all 44 genotypes evaluated in the study (Table 1). All amplifications were obtained without altering the recommended 
primer annealing temperature of the original loci. Amplification across the genus varied from $9 \%$ at locus CK007\% to $100 \%$ at loci CF48 and CF55 with the average amplification of loci similar for SSRs derived from C. florida (58\%) and C. kousa (55\%). For the SSRs derived from C. florida, 10 of 17 loci (CF20, CF48, CF51, CF55, CF59, CF105, CF122, CF124, CF125, and CF141) amplified in $60 \%$ or more of the species tested, whereas the SSRs derived from C. kousa amplified in $60 \%$ or more of the species tested for 11 of 19 loci (CK016, CK040, CK043, CK047, CK065, CK068, CK070, CK072, CK076, CK089, and CK089).

All C. florida derived SSR loci amplified in the three $C$. florida genotypes and the three interspecific hybrids genotypes with $C$. florida genetic background. Four of these loci (CF46, CF68, CF72, and CF100) only amplified in the C. florida individuals and interspecific hybrids, whereas the other 13 loci amplified Cornus species (C. angustata, C. capitata, $C$. elliptica, and C. kousa) native to Asia. Eleven C. florida SSR loci (CF20, CF46, CF47, CF66, CF68, CF72, CF100, CF105,
CF122, CF125, and CF597) only amplified in Cornus species from the big-bracted clade (C. angustata, $C$. capitata, $C$. elliptica, C. florida, C. kousa, C. kousa $\times$ C. florida, C. kousa $\times C$. nuttallii, and C. nuttallii). Two C. florida SSR loci (CF48 and CF124) amplified in at least one individual of the 18 Cornus species and hybrids tested. Loci CF48, CF51, CF55, CF59, and CF124 amplified in C. eyedena and C. mas.

All C. kousa SSR loci amplified DNA from genotypes from the big-bracted clade, whereas two loci (CK007 and CK071) amplified only C. kousa genotypes. No C. kousa SSRs amplified any of the $C$. eyedena or $C$. mas genotypes. The single $C$. alternifolia genotype amplified at loci CK016, CK070, CK076, and CK089, whereas the $C$. sericea genotypes only amplified at locus CK016.

The repeat type, allele size range, number of alleles, and the PIC for each locus are listed in Table 2. Alleles detected per locus varied from one (CK007) to 21 (CF105 and CF124) and the PIC values ranged from zero (CK007) to 0.93 (CF105 and CF124) with 23 of 36 loci having a PIC value of 0.80 or greater.

Table 2. Allelic information and polymorphism information content (PIC) of 36 Cornus simple sequence repeat loci tested for transferability in Cornus species and hybrids.

\begin{tabular}{|c|c|c|c|c|c|}
\hline Locus $^{\mathrm{Z}}$ & Genbank accession no. & Repeat motif & Allele size range (bp) & Alleles (no.) & $\mathrm{PIC}^{\mathrm{y}}$ \\
\hline CF20 & ED651708 & $(\mathrm{TC})_{22}$ & $136-166$ & 14 & 0.89 \\
\hline CF47 & ED651731 & $(\mathrm{AC})_{15}$ & $124-145$ & 9 & 0.79 \\
\hline CF51 & ED651735 & $\left.(\mathrm{AG})_{7 \cdot(\mathrm{GT}}\right)_{10}$ & $157-183$ & 17 & 0.88 \\
\hline CF55 & ED651738 & $(\mathrm{GT})_{7} \mathrm{~T}(\mathrm{TG})_{10}$ & $146-187$ & 17 & 0.92 \\
\hline CF59 & ED651742 & $(\mathrm{AAAG})_{4}$ & $77-365$ & 12 & 0.80 \\
\hline CF72 & ED651755 & $(\mathrm{AC})_{13}$ & $142-156$ & 4 & 0.49 \\
\hline CF100 & ED651777 & $(\mathrm{AC})_{14}$ & $116-130$ & 6 & 0.53 \\
\hline CF105 & ED651781 & $(\mathrm{TC})_{10}(\mathrm{AC})_{8}$ & $132-189$ & 21 & 0.93 \\
\hline CF122 & ED651798 & $(\mathrm{AAC})_{6}(\mathrm{AAT})_{3}$ & $94-122$ & 10 & 0.85 \\
\hline CF124 & ED651800 & $(\mathrm{TC})_{6} \cdot(\mathrm{CAAC})_{4}$ & $154-184$ & 21 & 0.93 \\
\hline CK007 & EU125522 & $(A G)_{8}$ & 108 & 1 & 0 \\
\hline CK015 & EU125523 & $(\mathrm{CT})_{10}$ & $94-140$ & 13 & 0.85 \\
\hline CK016 & EU821710 & $(\mathrm{CTG})_{4}$ & $147-153$ & 6 & 0.80 \\
\hline CK029 & EU125524 & $(\mathrm{TC})_{8}$ & $94-120$ & 8 & 0.66 \\
\hline CK031 & EU125525 & $(\mathrm{CT})_{7}$ & $112-139$ & 7 & 0.72 \\
\hline CK040 & EU125526 & $(\mathrm{GT})_{16}$ & $71-103$ & 9 & 0.79 \\
\hline CK043 & EU125527 & $(\mathrm{AT})_{6}$ & $91-121$ & 10 & 0.87 \\
\hline CK047 & EU125528 & $(\mathrm{AC})_{6}$ & $117-142$ & 8 & 0.84 \\
\hline CK048 & EU125529 & $(\mathrm{TA})_{6}$ & $148-174$ & 7 & 0.57 \\
\hline CK065 & EU821752 & $(\mathrm{GT})_{15}$ & $136-167$ & 12 & 0.87 \\
\hline CK066 & EU821753 & $(\mathrm{AC})_{11}$ & $142-188$ & 13 & 0.80 \\
\hline
\end{tabular}

${ }^{\mathrm{z} C F} 20-$ CF597 are simple sequence repeats (SSRs) isolated from Cornus florida and CK005-CK092 are SSRs isolated from C. kousa.

${ }^{y}$ Values range from 0 to 1.0 ; higher values indicate greater polymorphism. 
Sequence COMPARISON In CoRnus SPeCies. To determine if selected loci were present, selected Cornus species (indicated in Table 1 with an asterisk) were chosen and the PCR product obtained with CF48, CF59, and CF124, cloned, and sequenced. At locus CF48, the PCR products from 15 homozygous individuals consisting of 14 Cornus species (C. alba 'Aurea', C. alternifolia, C. amomum 'Walvatne', C. capitata 'Mountain Moon', C. elliptica, C. eyedena, C. florida 'Cherokee Brave', C. hessei 'Garden Glow', C. kousa 'Blue Shadow', C. mas 'Golden Glory', C. officinalis, C. pumila, C. racemosa, and C. sericea 'Cardinal') and one interspecific hybrid (C. kousa $\times C$. nuttallii 'Starlight') were cloned and sequenced and the 14 species were successfully sequenced. For locus CF59, the PCR products from three heterozygous individuals (C. mas 'Golden Glory', C. mas 5-2, and C. mas 25) and 10 homozygous individuals ( $C$. alternifolia, $C$. capitata, C. elliptica, C. eyedena, C. florida 'Cherokee Brave', C. kousa 'Blue Shadow', $C$. kousa $\times$ C. florida 'Ruth Ellen', C. kousa $\times$ C. nuttallii 'Starlight', C. nuttallii 'Colrigo Giant', and C. officinalis) were cloned and sequenced. Seven PCR products ( $C$. florida 'Cherokee Brave', C. kousa 'Blue Shadow', both C. mas 'Golden Glory', one each for C. mas 5-2 and C. mas 25, and C. nuttallii 'Colrigo Giant') were successfully cloned and sequenced for locus CF59. For locus CF124, the PCR products from 17 homozygous individuals (C. alba 'Aurea', C. alternifolia, $C$. amomum 'Walvatne', C. capitata 'Mountain Moon', C. elliptica, C. eyedena, C. florida 'Cherokee Brave', C. hessei 'Garden Glow', C. kousa 'Blue Shadow', C. kousa $\times$ C. florida 'Ruth Ellen', C. kousa $\times$ C. nuttallii 'Starlight', C. mas 'Golden Glory', C. nuttallii 'Colrigo Giant', C. pumila, C. racemosa, C. sericea 'Cardinal' and yellow twig accession) were cloned and sequenced. The only PCR products for which sequencing was unsuccessful for locus CF124 were C. kousa 'Blue Shadow', $C$. kousa $\times$ C. florida 'Ruth Ellen', and C. racemosa.
The sequencing assay carried out in the selected individuals (data shown for locus CF48 only) for the loci resulted in the presence of conserved primer sites and the expected repeat motif in all successful sequencing reactions with the exception of the second allele amplified by locus CF59 (C. mas genotypes only). For these samples, the larger allele did not contain the SSR motif, whereas the smaller allele did contain the expected SSR motif. It should also be noted that all of the nonbig-bracted Cornus species tested, other than the three $C$. mas genotypes sequenced, with locus CF59 showed a faint PCR product within the same range as the heterozygous individuals at this locus. When these samples were reamplified with DMSO added to the PCR reaction, the faint PCR products and stutter bands exhibited in all of the nonbig-bracted Cornus species were absent.

The SSR motifs tended to be shorter in non- $C$. florida species than in $C$. florida from which the SSRs were first isolated, although this was not the case for locus CF124 (data not shown). In addition to variations in repeat length, the SSRs examined can be summarized with locus CF48, whose aligned sequences are shown in Figure 1.

The forward primer site sequence for CF48 was completely conserved for all species, whereas the reverse primer site had two modifications and the $(\mathrm{GT})_{9}$ repeat found in $C$. florida, the source species, was shorter in all other species evaluated. Several single-base substitutions were revealed in multiple species at three sites in the repeat. For example, a $\mathrm{T} \rightarrow \mathrm{C}$ substitution occurred in $C$. officinalis and $C$. mas 'Golden Glory', a $\mathrm{G} \rightarrow \mathrm{A}$ substitution in $C$. kousa 'Blue Shadow', $C$. capitata 'Mountain Moon', C. officinalis, C. mas 'Golden Glory', and $C$. eyedena, a $\mathrm{G} \rightarrow \mathrm{A}$ substitution in $C$. hessei 'Garden Glow', C. alba 'Aurea', C. sericea 'Cardinal', and C. alternifolia.

Twenty-one point mutations were present in the flanking region of locus CF48 and the informative variations are

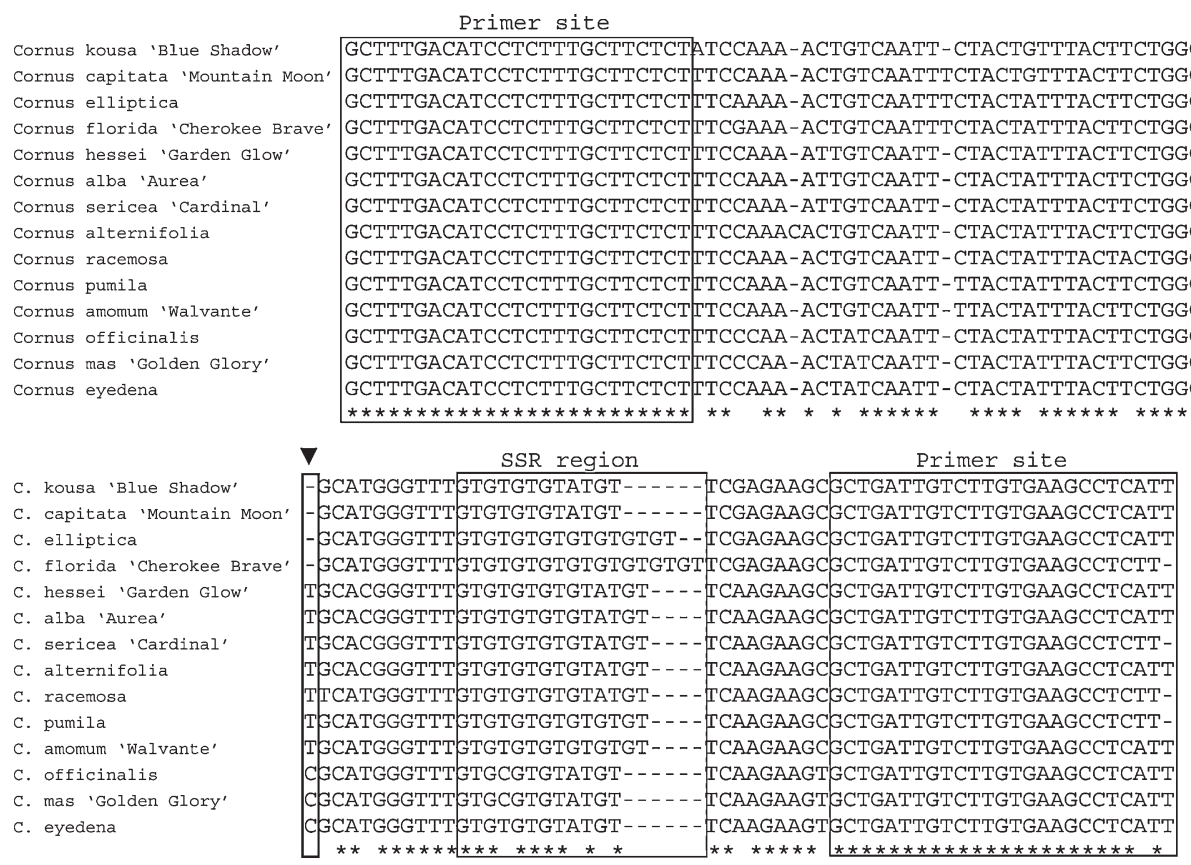

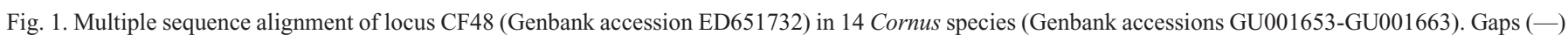
have been placed to maximize the homology, asterisks $(*)$ correspond to sequence positions with identical nucleotides in all the species. Primer sites and simple sequence repeats are indicated with boxes labeled, respectively. Arrows above the boxes indicate informative mutation sites. 
indicated with arrows in Figure 1. At mutation sites 1 and 2, the blue- and white-fruited phenotypes had a $\mathrm{T} \rightarrow \mathrm{G}$ substitution followed by an $\mathrm{A} \rightarrow \mathrm{T}$ substitution. There was a single insertion of a $\mathrm{T}$ in the blue- and white-fruited phenotypes at both sites and a single insertion of a $\mathrm{C}$ at both sites in the cornelian cherries phenotypes at mutation sites 3 and 4 .

The mutation sites in the reverse primer site, repeat motif, and the regions flanking the SSR were analyzed phylogenetically to group species according to presumed ancestry. Maximum parsimony analysis recovered a single tree of 23 steps that was identical topologically with the inferred Bayesian majority rule consensus tree (Fig. 2). The inferred tree was rooted manually based on results published by Xiang et al. (2006). The tree showed three main clades, each including species with similar phenotypes. Specifically, the big-bracted dogwoods grouped together with the Asian species branching from lone species from North America. The three cornelian cherries grouped together and the blue- and white-fruited dogwood species formed a sister group to the cornelian cherries.

\section{Discussion}

Transfer SuCCESS OF C. FLORIDA AND C. KOUSA DOGWOOD SIMPLE SEQUeNCE REPEATS ACROSS CORNACEAE. SSRS are

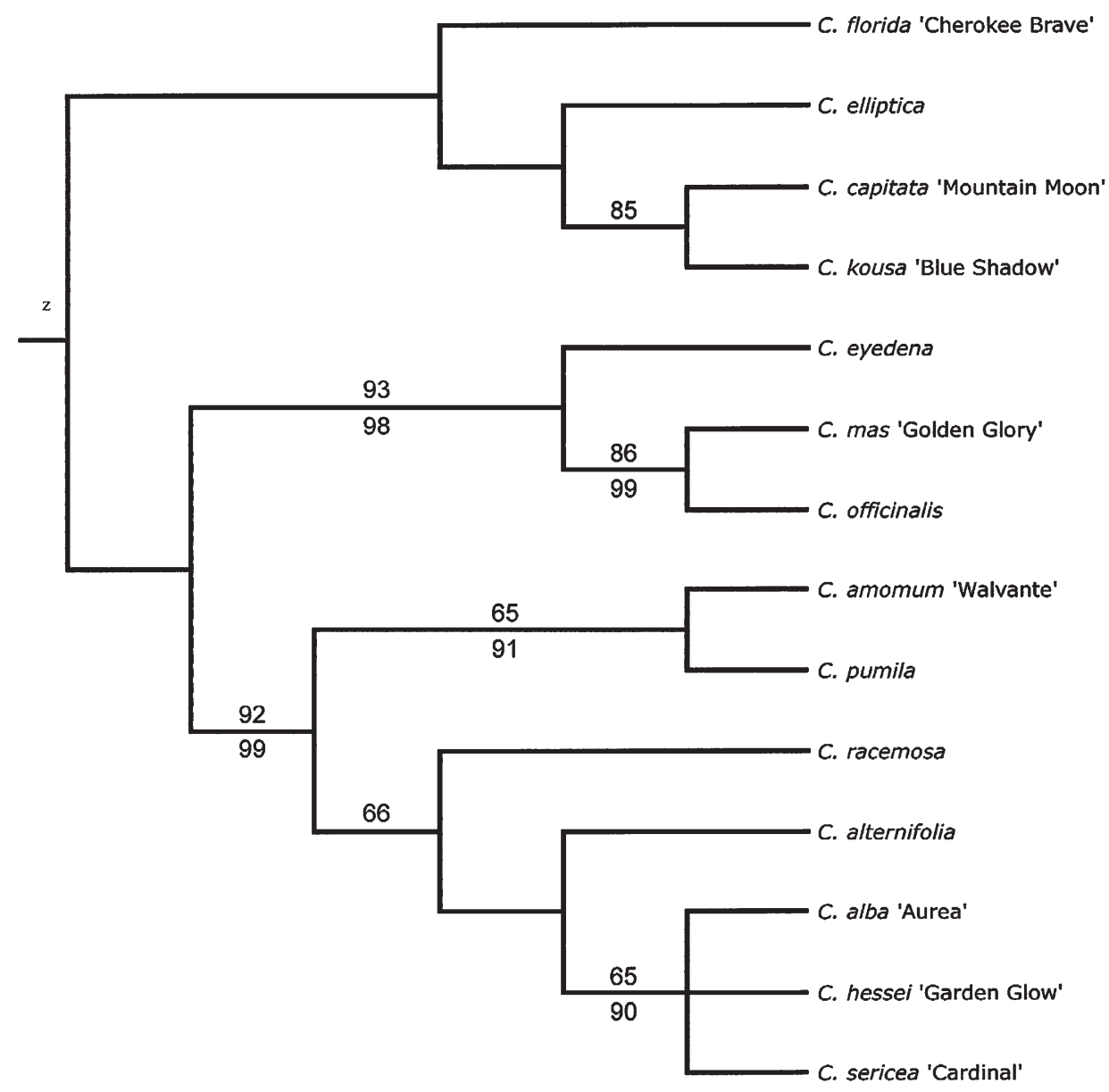

Fig. 2. Most parsimonious tree (length $=23$ steps) inferred from simple sequence repeat sequences obtained from 14 Cornus species. Bootstrap scores and Bayesian posterior probabilities shown above and below each node

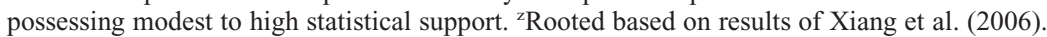

thought to occur less frequently in the genomes of plants than in genomes of other organisms (Powell et al., 1996). When this is combined with the cost and expertise needed to isolate new SSR loci, the possibility of cross-species transferability of SSRs from closely related nonsource species becomes advantageous. It is common to modify PCR protocols for successful crossspecies transferability of SSRs. Modification of PCR protocols was necessary in $65 \%$ of the studies testing cross-species transferability of SSRs reviewed by Rossetto (2001). In our study, no modifications were made to our PCR protocol for successful cross-species transfer. SSRs isolated from one species may be useful in genetic studies on other closely related species with amplification of SSR loci ranging from $50 \%$ to $100 \%$ between species within genera in plants (Peakall et al., 1998). The benefits of cross-species transferability of SSRs was reviewed by Rossetto (2001), who found that closely related species are more likely to share SSR priming sites than distantly related species and when there is cross-species transferability, high levels of polymorphism exist.

The conservation of primer sites is necessary for the amplification of SSR loci in species other than the source of the SSRs. Transferability of SSR loci isolated from C. florida to the other species and hybrids from the genus was higher than the transferability of SSR loci isolated from C. kousa, providing evidence that primer sites and flanking regions are conserved across Cornus. Our results of crossspecies transferability in Cornus are consistent with those of Keir (2008). In that study, SSRs from C. florida developed by Cabe and Liles (2002) were successfully used to conduct a population genetics study of $C$. nuttallii.

Another way to measure the usefulness of an SSR locus is the PIC value, because markers with higher PIC values are more informative and useful for molecular studies (Fernández et al., 2008). Overall, SSR loci isolated from $C$. florida exhibited higher polymorphism (mean 0.77 PIC value) as compared with loci isolated from C. kousa (mean 0.70 PIC value). The frequency of the most common allele in some of the loci (CF141, CK016, CK089) and the low number of alleles detected with other loci (CF72, CK007, CK071) could be reasons for high and low PIC values found in this study. Still, the high level of SSR polymorphism could be attributed to Cornus species being obligate outcrossing species that are considered self-sterile (Gunatilleke and Gunatilleke, 1984; Hummel et al., 1982; Orton, 1985; Reed, 2004; Sork et al., 2005; Wadl et al., 2009).

Amplification of PCR products across species indicate that primer sites are highly conserved and does 
not always guarantee the presence of the specific repeat, which is required for using SSRs as molecular markers in nonsource species. To determine if homologous loci are present in nonsource species, it is necessary to verify the PCR products by sequencing because product size and variation are not always indicators of cross-species transferability of SSRs (Rossetto, 2001). This same author notes that stutter bands are good indicators of the presence of SSRs. Although we observed stutter bands in some of the loci tested in this study, but not uniformly across the study, to confirm presence of a repeat, we sequenced three C. florida SSR loci (CF48, CF59, and CF124) that amplified in all of the species sampled in this study. When DMSO was included in the PCR reaction, stutter bands were absent. Additives such as DMSO can be added to the PCR or sequencing reaction to increase yield, specificity, and consistency (Varadaraj and Skinner, 1994; Winship, 1989). We found that the primer sites and flanking regions were highly conserved and that repeat was present for all of the individuals sequenced. However, for loci CF48 and CF59, the repeat was longer in the source species than in the nonsource species, which is in agreement with the findings of Peakall et al. (1998), whereas the repeat for locus CF124 was longer in a nonsource species. Our sequencing results support those of Keir (2008), which demonstrated that SSR loci repeats isolated from C. florida were present in individuals of $C$. nuttallii in addition to other nonsource species.

Previously, a comprehensive species-level phylogeny of Cornus was constructed using ITS and matK sequences and four major clades (blue- or white-fruited, cornelian cherries, big-bracted, and dwarf) were delineated for the genus (Xiang et al., 2006). Based on the sequences used in this study, the bigbracted and dwarf clades are sister clades with the cornelian cherries being sisters to these two clades, whereas the blue- or white-fruited clade is sister to the other clades. The SSR loci used in this study were isolated from two closely related species (C. florida and C. kousa) belonging to the big-bracted clade. Our study consisted of species that were delineated to three (blue- or white-fruited, cornelian cherries, and big-bracted) of the four clades. The phylogenetic tree reported in Figure 2 confirms the results of Xiang et al. (2006) and demonstrates that SSRs developed from one Cornus species can be used to delineate closely related species in the genus.

Our results indicate that previously developed SSR loci from one species can be potentially used in future breeding, population, ecological, and genetic studies of other species within Cornus as demonstrated by the following: 1) SSR primers developed from one species can be used to amplify homologous loci of related species; 2) cross-species transferability of SSR loci results from conservation of primer sites; and 3) SSR primers have potential for Cornus phylogenetic analyses. With over 900 publicly available SSR loci available for C. florida and C. kousa (Cabe and Liles, 2002; Wadl et al., 2008; Wang et al., 2008), dogwood SSRs have enormous potential to provide genetic tools for investigations of various Cornus species.

\section{Literature Cited}

Anderson, J.A., G.A. Churcill, J.E. Autrique, S.D. Tanksley, and M.E. Sorrels. 1992. Optimizing parental selection for genetic linkage maps. Genome 36:181-186.

Bassam, B.J., G. Caetano-Anollés, and P.M. Greshoff. 1991. Fast and sensitive silver staining of DNA in polyacrylamide gels. Anal. Biochem. 196:80-83.
Cabe, P.R. and J.S. Liles. 2002. Dinucleotide microsatellite loci isolated from flowering dogwood (Cornus florida L.). Mol. Ecol. Notes 2:150-152.

Conduit, R. and S.P. Hubbell. 1991. Abundance and DNA sequence of two-base repeat regions in tropical tree genomes. Genome 34:66-71.

Felsenstein, J. 1985. Confidence limits on phylogenies: An approach using the bootstrap. Evolution 39:783-791.

Fernández, M.P., Y. Núñez, F. Ponz, S. Hernáiz, F.J. Gallego, and J. Ibáñez. 2008. Characterization of sequence polymorphisms from microsatellites flanking region in Vitis spp. Mol. Breed. 22:455-465.

Gunatilleke, C.V.S. and I.A.U.N. Gunatilleke. 1984. Some observations on the reproductive biology of three species of Cornus (Cornaceae). J. Arnold Arbor. 65:419-427.

Hamada, H., M.G. Petrino, and T. Kakunaga. 1982. A novel repeated element with Z-DNA-forming potential is widely found in evolutionarily diverse eukaryotic genomes. Proc. Natl. Acad. Sci. USA 79:6465-6469.

Hummel, R.L., P.D. Ascher, and H.M. Pellett. 1982. Genetic control of self-incompatibility in red-osier dogwood. J. Hered. 73:308-309.

Jones, R.L. 2005. Plant life of Kentucky-An illustrated guide to the vascular flora. Univ. of Kentucky Press, Lexington, KY.

Keir, K.R. 2008. Beautiful but lacking diversity: Population genetics of Pacific dogwood (Cornus nuttallii Audobon ex Torr. \& A. Gray). MS Thesis, Univ. of British Columbia, Vancouver, BC, Canada.

Kijas, J.M.H., J.C.S. Fowler, and M.R. Thomas. 1995. An evaluation of sequence tagged microsatellite site markers for genetic analysis within Citrus and related species. Genome 38:349-355.

Larkin, M.A., G. Blackshields, N.P. Brown, R. Chenna, P.A. McGettigan, H. McWilliam, F. Valentin, I.M. Wallace, A. Wilm, R. Lopez, J.D. Thompson, T.J. Gibson, and D.G. Higgins. 2007. Clustal W and Clustal $X$ version 2.0. Bioinformatics 23:2947-2948.

Mitchell, W.A., P.A. Gibbs, and C.O. Martin. 1988. Flowering dogwood (Cornus florida). U.S. Army Corps Eng. Wildlife Res. Mgt. Manual Tech. Rpt. EL-88-9.

Orton, E.R., Jr. 1985. Interspecific hybridization among Cornus florida, C. kousa, and C. nutallii. Proc. Intl. Plant Prop. Soc. 35:655-661.

Peakall, R., S. Gilmore, W. Keys, M. Morgante, and A. Rafalski. 1998. Cross species amplification of soybean (Glycine max) simple sequence repeats (SSRs) within the genus and other legume genera: Implications for the transferability of SSRs in plants. Mol. Biol. Evol. 15:1275-1287.

Posada, D. and K.A. Crandall. 1998. ModelTest: Testing the model of DNA substitution. Bioinformatics 14:817-818.

Powell, W., G.C. Machray, and J. Provan. 1996. Polymorphism revealed by simple sequence repeats. Trends Plant Sci. 1:215-222.

Reed, S.M. 2004. Self-incompatibility in Cornus florida. HortScience 39:335-338.

Ronquist, F. and J.P. Huelsenbeck. 2003. MrBayes 3: Bayesian phylogenetic inference under mixed models. Bioinformatics 19:1572-1574.

Rossetto, M. 2001. Sourcing of SSR markers from related plant species, p. 211-224. In: R.J. Henry (ed.). Plant genotyping-The DNA fingerprinting of plants. CABI, New York, NY.

Sork, V.L., P.E. Smouse, V.J. Apsit, R.J. Dyer, and R.D. Westfall. 2005. A two-generation analysis of pollen pool genetic structure in flowering dogwood, Cornus florida (Cornaceae), in the Missouri Ozarks. Amer. J. Bot. 92:262-271.

Swofford, D.L. 2002. PAUP*: Phylogenetic analysis using parsimony (* and other methods), Version 4.0b 10. Sinauer Associates, Sunderland, MA.

Thomas, M.R. and N.S. Scott. 1993. Microsatellite repeats in grapevine reveal DNA polymorphisms when analyzed a sequence-tagged sites (STSs). Theor. Appl. Genet. 86:985-990.

Tural, S. and I. Koca. 2008. Physico-chemical and antioxidant properties of cornelian cherry fruits (Cornus mas L.) grown in Turkey. Scientia Hort. 116:362-366.

Varadaraj, K. and D.M. Skinner. 1994. Denaturants or cosolvents improve the specificity of PCR amplification of a G+C-rich DNA using genetically engineered DNA polymerases. Gene 140:1-5. 
Wadl, P.A., X. Wang, B.E. Scheffler, T.A. Rinehart, and R.N. Trigiano. 2008. Microsatellites from kousa dogwood (Cornus kousa). Mol. Ecol. Resources 8:780-782.

Wadl, P.A., J.A. Skinner, J.R. Dunlap, T.A. Rinehart, S.M. Reed, V.R. Pantalone, and R.N. Trigiano. 2009. Honeybee-mediated controlled pollinations in Cornus florida and C. kousa intra- and interspecific crosses. HortScience 44:1527-1533.

Wang, X., R. Trigiano, M. Windham, B. Scheffler, T. Rinehart, and J. Spiers. 2008. Development and characterization of simple sequence repeats for flowering dogwood (Cornus florida L.). Tree Genet. Genomes 4:461-468.

Wang, X.W., R.N. Trigiano, M.T. Windham, R.E. DeVries, B.E. Scheffler, T.A. Rinehart, and J.M. Spiers. 2007. A simple PCR procedure for discovering microsatellites from small insert libraries. Mol. Ecol. Notes 7:558-561.
Winship, P.R. 1989. An improved method for sequencing PCR amplified material using dimethyl sulphoxide. Nucleic Acids Res. 17:1266.

Wu, K.S. and S.D. Tanksley. 1993. Abundance, polymorphism and genetic mapping of microsatellites in rice. Mol. Gen. Genet. 241: 225-235.

Xiang, Q.Y., D.T. Thomas, W. Zhang, S.R. Manchester, and Z. Murrell. 2006. Species level phylogeny of the genus Cornus (Cornaceae) based on molecular and morphological evidence-Implications for taxonomy and tertiary intercontinental migration. Taxon 55: 9-30.

Yilmaz, K.U., S. Ercisli, Y. Zengin, M. Sengul, and E.Y. Kafkas. 2008. Preliminary characterisation of cornelian cherry (Cornus mas L.) genotypes for their physico-chemical properties. Food Chem. 114: 408-412. 